\title{
An Optical Sensor System for Characterization of Ceramics based on Laser Speckle Photometry
}

\author{
Lili Chen ${ }^{1}$, Ulana Cikalova ${ }^{1}$, Beatrice Bendjus ${ }^{1}$, Stefan Muench', Mike Roellig ${ }^{1}$ \\ ${ }^{1}$ Fraunhofer Institute for Ceramic Technologies and Systems IKTS, Maria-Reiche-Straße 2, 01109 \\ Dresden, Germany
}

\begin{abstract}
Advanced ceramic components are widely used in the industrial applications, e.g. substrates of power electronic modules, and electrolytes of new generation batteries. As a brittle material, the crack propagation is very sensitive to the stress distribution of ceramics. Existing defects under extreme stress condition will lead to the functional failures of the whole component. In order to avoid the crack initiation and propagation, and guarantee a good quality state of ceramic products, stress condition and defects should be monitored and detected.

In this paper, an optical non-destructive testing method - Laser Speckle Photometry (LSP) will be demonstrated to determine mechanical stresses in ceramic substrates and detect defects in ceramic products for the industrial applications. The LSP method is based on the analysis of a time resolved speckle dynamics which is activated by an external excitation. The speckle signal can be correlated to the external introduced signal and further be related to our targeting parameters - mechanical stress and existing defects in the ceramics. The preliminary results of LSP has shown its great potential for non-destructive characterization of ceramics in terms of stress monitoring and defect detection.
\end{abstract}

Keywords: Optical sensor system, Laser speckle photometry, Non-destructive testing, Strain/stress characterization, defects detection in ceramics

\section{Introduction}

Due to the special structural and functional properties, the advanced ceramic technology plays an important role in many fields of the industry. For example, direct copper bonding (DCB) substrates are widely used in electronic applications because of its high thermal conductivity performance, and Na-ß-Alumina can be utilized to produce high-temperature, low-cost batteries. During the manufacturing process or under the service loading, high thermal or mechanical stress would be occurred. Extreme stress condition might lead to the crack initiations. The propagation of cracks in ceramic components will result in security risks and great financial losses for manufacturers and customers. In order to ensure a continuously high quality of ceramic products in the industrial applications, it is important to know exactly the stress distribution and be able to detect the existing defects on ceramics. Currently, there is no technique that allows direct, non-contact and fast determination of mechanical stresses in ceramics. Techniques such as X-ray diffraction method, the digital speckle pattern interferometry, and ultrasonic scanning microscopy are time-consuming, very expensive and sometimes destructive. For the defect detection of ceramics are common used methods like X-ray transmission and backscattering techniques, ultrasound reflectance, with the aim of detecting defects in near surface zone as well as inner zone. These procedures are usually complicated, and the sample are individually scanned by laboratory equipment. It is difficult to integrate the devices into production process. However, there is a high economic demand for the integration of inspection setups into production lines in order to locally verify important quality parameters and to detect defects as well. Component failures in the application will result in security risks and great financial losses for manufacturers and customers.

In this case, the authors propose a novel nondestructive and non-contact optical technique Laser Speckle Photometry (LSP) for the characterizations of ceramics including stress monitoring and defect detection in a fast and cost effective way.

\section{Laser Speckle Photometry}

A speckle pattern is generated when an optical rough surface illuminated by a coherent light source, e.g. laser [1]. The scattered waves from various points of the illuminated surface 
interfere in the observation plane, generating the speckle pattern, i.e. a spatial structure with randomly distributed intensity minima and maxima. Different luminosities can be recorded by a CCD and CMOS chip respectively. The theory of speckle or dynamic speckle belongs to the category of statistical optics of which a detailed account of phenomena is given by Goodman [2] including the mathematical description of speckle properties, for instance the central limit theorem of probability theory, random processes. The LSP method is based on the analysis of speckle patterns generated by a laser source. Actually the recorded speckle patterns are not only 2-D graphics, but also the fingerprint containing the $3-D$ information of sample surface. The basic experimental setup of LSP contains three parts - an illumination source, an external excitation source and a detector, as shown in Fig. 1. A laser diode as the illumination source generate a static speckle pattern on sample surface. Speckles can be activated by an external thermal or mechanical excitation to yield a time resolved dynamic speckle pattern, which can be also called speckle movement. Thanks to the development of hardware and mathematical tools for registration and processing of non-stationary optical fields, the time resolved speckle patterns can be recorded by a digital camera and be evaluated with different algorithms for different specific applications.

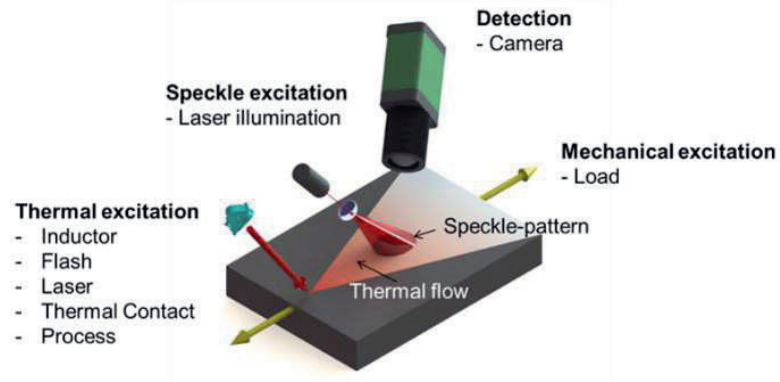

Fig. 1: Schematic structure of Laser Speckle Photometry.

Generally, the LSP technique can be used for characterization of a material's defects [3]. It provides a solution of fast in-line defect inspection in industrial field. Much work has been done by LSP method for metallic materials including the characterization of material properties, for example, damage, hardness and porosity [3-5]. Since ceramic materials have total different thermal and mechanical properties compared with metals, characterization of ceramics based on LSP show its special research values in both academic and industrial fields.

\section{Stress characterization of ceramics by LSP}

In the research of stress characterization, the strain field was introduced by mechanical tension with a 3-point-bending device. Rough surfaces of ceramic samples respond to this stimulation with changing the position of surface reflectors respectively scattering points and converting the variations into changes of speckle pattern recorded by the camera system in form of video sequences.

In order to connect the speckle signal to mechanical signal, a calibration function of speckle and stress should be established. A concept of the calibration was developed which describes the correlation between both variables, and the details were presented in [6]. Briefly, in one hand, the process of timeresolved dynamic speckle is led by surface strain changes introduced by the bending test. In the other hand, the behavior between mechanical stress and strain can be described by the Hooke's law in the elastic region and for small stretches. Therefore, strain is a transfer parameter connecting the speckle and mechanical signals. Since the temperature of sample was controlled in a constant during the measurement, the surface change caused by the thermal properties (e.g. thermal expansion) was not taken into consideration any more.

As mentioned above the LSP signal is dependent on the surface strain of sample, in that case, the mechanical strain can be denoted by the speckle signal $S_{L S P}(t)$, the calibration function is given by:

$$
\sigma(t)=P_{1} * S_{L S P}(t)+P_{2}
$$

where $P_{1}$ and $P_{2}$ should be determined by the experimental calibration test. Because the initial state of sample was defined to be stressless, the parameter $P_{2}$ was set to zero. $P_{1}$ could be estimated by linear fitting of mechanical stress computed by finite element method (FEM) simulation from the calibration measurement and the LSP signal $S_{L S P}(t)$. After the determination of parameter $P_{1}$, the real stress condition of ceramic sample can be calculated directly from LSP signal based on the calibration function (1) [6].

\section{Defect detection of ceramics by LSP}

As it is mentioned above, change of surface strain can activate dynamic speckle patterns. And the strain change could also be produced by the change of temperature, i.e. thermal expansion. When ceramic sample is illuminated by an external thermal source, e.g. a high power laser, the thermal energy will be absorbed by the sample, and it will propagate on the sample surface, which results in the local temperature differences. The change of temperature causes 
strain change on sample surface activating time resolved dynamic speckle patterns, which is shown in Fig. 2.

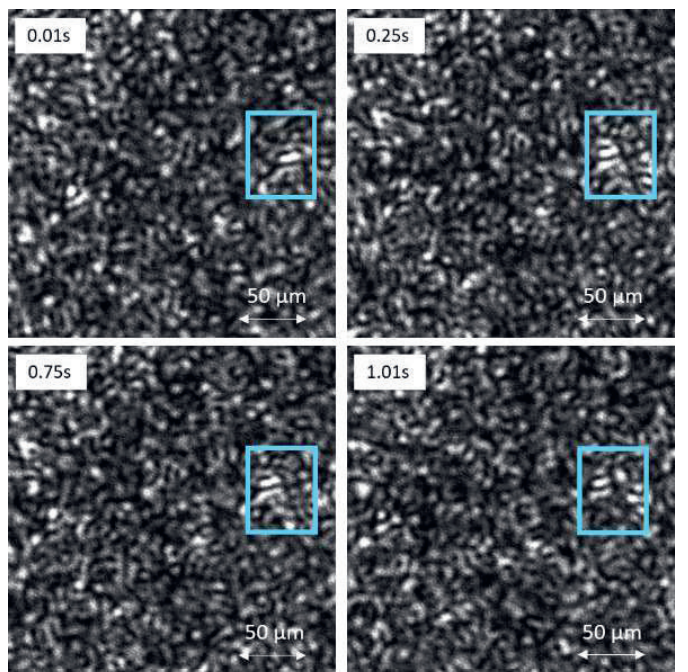

Fig. 2: Time-resolved speckle patterns.

When thermal waves propagate on an undefective sample surface, a homogeneous spatial gradient of temperature will be formed. If surface defects exist, the local magnitude of the thermal propagation will be amplified, i.e. the presence of defects change distribution of thermal energy around the defective area [7]. To be exact, the mentioned local change of heat distribution results in the thermal energy accumulating around the defective area, as presented in Fig. 3.

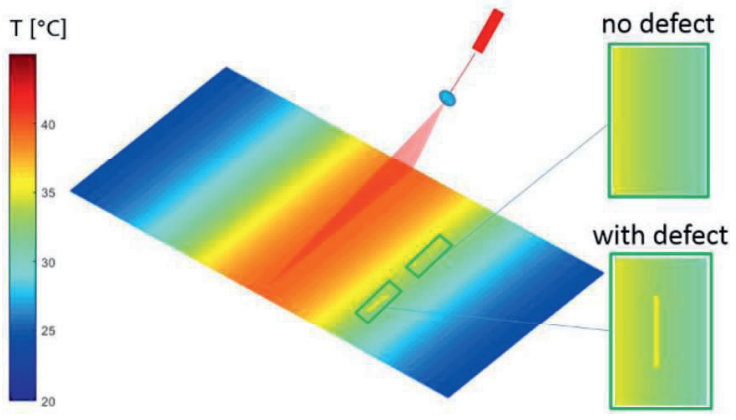

Fig. 3: Simulation of thermal propagation on a defective surface.

The thermal accumulation distorts the normal spatial gradient and leads to a higher level of speckle movement at the border of defects compared with the surroundings. As a result, a contrast of dynamic speckle movement between defective area and background could be obtained. On the basis of special algorithms, dynamic speckle signal can be evaluated and analyzed to extract the contrast, which provides the possibility for defect detection by the technique of LSP.

Unlike other speckle based inspection techniques, for example, speckle interferometry and shearography, the LSP works without reference beams. This allows a simple but robust construction, and last but not least, measurement and evaluation of obtained data could be performed in real time. Therefore, it can be easily integrated into the manufacture process to implement the in-line inspection.

\section{Algorithms}

There are two basic evaluation methods of LSP, static and quasi-static algorithms, to evaluate speckle signals in the different situations. The static algorithm focuses on the image scale, which uses the gray value related parameters combined with statistical properties of speckle recordings. Parameters, for instance, mean value of intensity, speckle contrast, speckle size and texture properties calculated from the greylevel co-occurrence matrix (GLCM) $[8,9]$ can be utilized to present speckle signals in LSP technique. In the quasi-static evaluation algorithms, the differences of gray value distribution of a single pixel in time sequence rather than image scale are interested. In our research of characterization of ceramics including stress characterization and defect detection, quasi-static algorithms were used to calculate the speckle parameters for final results.

For the purpose of stress characterization, an algorithm based on correlation method was proposed. In the field of image processing, the correlation can be explained by another word, i.e. similarity. A high correlation means a great similarity of two different signals. In [10], strain information of a brass plate in tensile test was calculated by one dimensional speckle correlation. And microscopic deformations of the uniform tensile test specimen were measured by digital speckle correlation in [11]. Taking into account the characteristics of the speckle pattern generated by a laser source on the surface of ceramic materials, a parameter based on correlation techniques was selected to present speckle signals in the correlation function. The parameter is 2-D correlation coefficient whose mathematical expression is shown in equation (2).

$$
r(t)=\frac{\sum_{i} \sum_{j}\left(I_{i j}\left(t_{0}\right)-\overline{I_{0}}\right)\left(I_{i j}(t)-\bar{I}_{t}\right)}{\sqrt{\left(\sum_{i} \sum_{j}\left(I_{i j}\left(t_{0}\right)-\overline{I_{0}}\right)^{2}\right)\left(\sum_{i} \sum_{j}\left(I_{i j}(t)-\overline{I_{t}}\right)^{2}\right)}}
$$

The coefficient $r(t)$ was renamed by correlation coefficient of speckles (CCoS), which presents the similarity between two recorded speckle images. In equation (2), $I_{i j}\left(t_{0}\right)$ is gray value of pixel $(i, j)$ in speckle image at the stressless state, which is the first frame of speckle recording. And $\overline{I_{0}}$ means the average gray value of all pixels in the first frame. Analogously, 
$I_{i j}(t)$ and $\overline{I_{t}}$ present the intensity of pixel $(i, j)$ in speckle images at different stress states and the average intensity of corresponding frames. In one speckle recording, the value of CCoS demonstrates the change of time-resolved speckle patterns in a specific frame compared with the first frame, which reveals the changes of stress in tested samples.

In the research of defect detection, the algorithm was based on a time-resolved analysis shown in Fig. 4. The intensity of each pixel was processed in the time domain. During the movement of speckle patterns, the timevaried intensity can be related to the spatial gradient of temperature with a special correlation function. It has been proven in the research of Kazak [12] that the value of the correlation function was proportional to the local temperature of a certain region of interest. As distinguished from the CCoS mentioned above, the correlation function used in the defect detection calculated the intensity differences of pixels in the speckle recordings. And it is given by:

$$
C(\tau)=\langle I(1+\tau, x, y)-I(1, x, y)\rangle^{2}
$$

where $\tau$ is the time shift or the number of frame intervals, $I(n, x, y)$ is the intensity of pixel whose location is determined by the coordinates $\mathrm{x}$ and $y$ in the $n$-th frame. This correlation function is modified from the so-called semivariogram, which is a geostatistical tool for studying the relationship between collected data as a function of distance and direction [13].

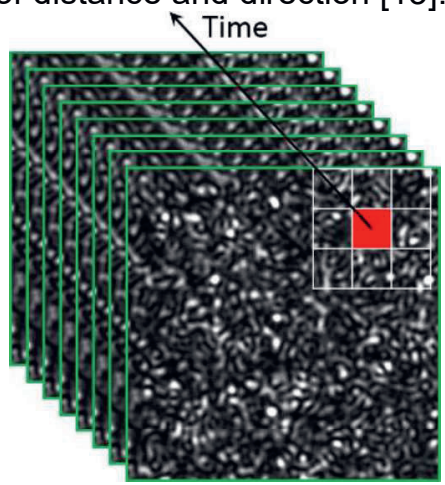

Fig. 4: Schematic of the time-resolved image analysis.

The relationship between the value of correlation function and the change of local temperature is shown in Fig. 5. From the diagram, it can be concluded that before saturation point, the numerical value of correlation function exhibits similar behavior to that of the local temperature, which is entirely consistent with the conclusion of Kazak mentioned above.

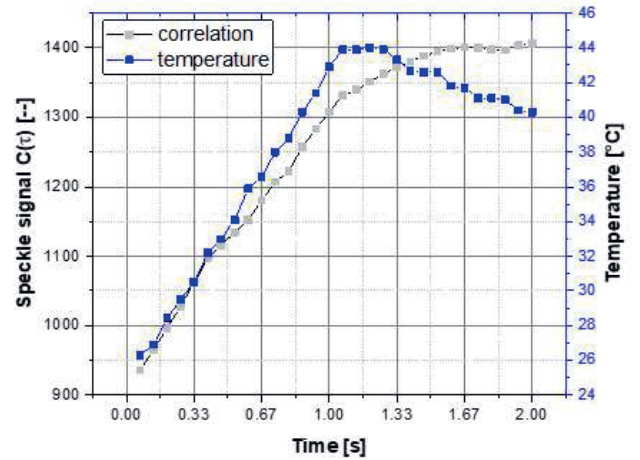

Fig. 5: relationship between correlation function and local temperature.

As it is known, an image can be demonstrated by a two-dimensional matrix in the digital processing. Similarly, the video file recording the speckle signals can be treated as a threedimensional matrix. The time-varied intensity of each pixel is a dimensionless signal. Since the thermal energy would be accumulated around defective area, the dynamic speckle movement is stronger than that of background. Therefore, frequency analysis could be utilized to extract the contrast of dynamic speckle signals between defective area and background. After the calculation of correlation function for each pixel, the Short Time Fourier Transformation (STFT) or Wavelet Transformation (WT) is applied to convert the dimensionless signal into time-frequency domain $[14,15]$. In this case, the amplitudes of STFT or WT for all pixels at a specific time-frequency point can be selected to build the resulting image.

\section{Experiments and results}

\section{Stress characterization of ceramics}

The experimental setup of calibration measurements combined the optical setup for LSP recordings and the mechanical configuration for stress and strain introduction. The setup is shown in Fig. 6. The speckle patterns were generated on sample surface by a single mode laser diode with a wavelength of $650 \mathrm{~nm}$. The diameter of total illumination area was approximately $8 \mathrm{~mm}$. Dynamic speckle signals were recorded by a CMOS digital camera, whose full resolution of the chip was $2048{ }^{*} 1088$ pixels. A macro lens with 8 times magnification was mounted on the camera. The beam splitter provided the possibility to make both camera and laser diode be perpendicular to sample surface. In this case, during the bending test there is no out-of-plan motion at the measuring position. It ensured that the dynamic speckle patterns were generated only by the change of surface strain, which reduced the noise coming from the setup in final results. 
Samples were fixed in a 3-point bending device. The distance between the outer loading pins was $40 \mathrm{~mm}$. The samples under test were a thin $\mathrm{Al}_{2} \mathrm{O}_{3}$ plate $\left(45^{*} 10^{*} 0.62 \mathrm{~mm}^{3}\right)$ and a thin low temperature co-fired ceramic (LTCC) plate $\left(45^{\star} 10^{*} 0.67 \mathrm{~mm}^{3}\right)$. The measurements occurred at the center of sample surfaces. During the bending measurement, surface strain was introduced into sample via the displacement of loading pins of the device, which was under controlled. The speckle recordings were evaluated by means of the software MATLAB ${ }^{\circledR}$. Meanwhile the mechanical stress at the measuring position was estimated by FEM simulations.

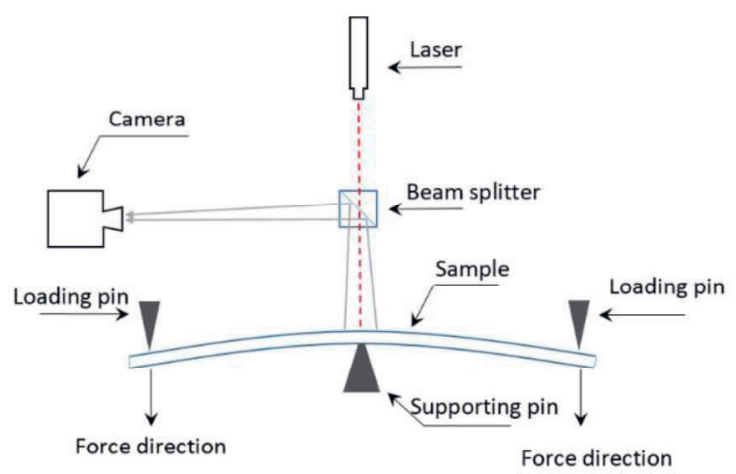

Fig. 6: Experimental setup of LSP for stress characterization.

The preliminary results of calibration measurements are shown in Fig. 7 (a-c). The data were obtained from a $200 * 200 \mu \mathrm{m}^{2}$ area on sample surface. The stress was estimated from FEM simulation, and the speckle parameter CCoS was calculated based on a quasi-static algorithm, i.e. equation (2) mentioned above. The measurements were repeated 4 times at same position for each sample. Both variables were plotted against the time of measurement in the diagrams.

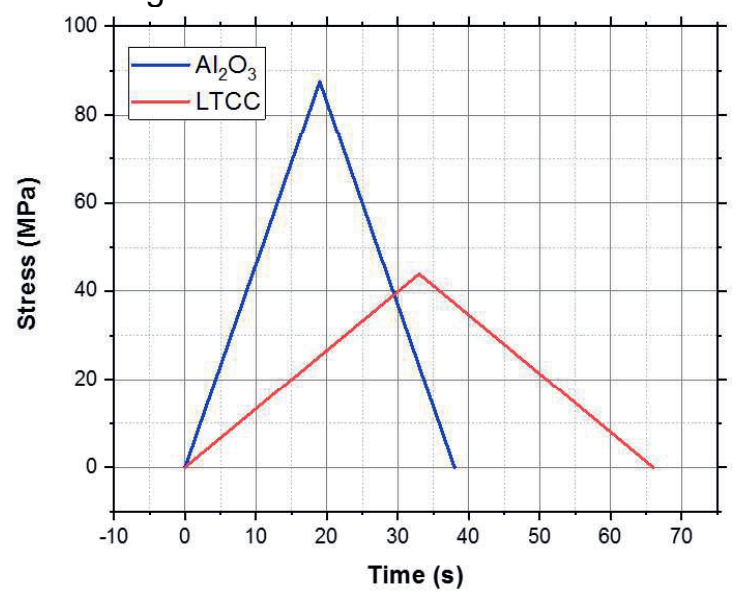

Fig. 7(a): Stress signals calculated from FEM.

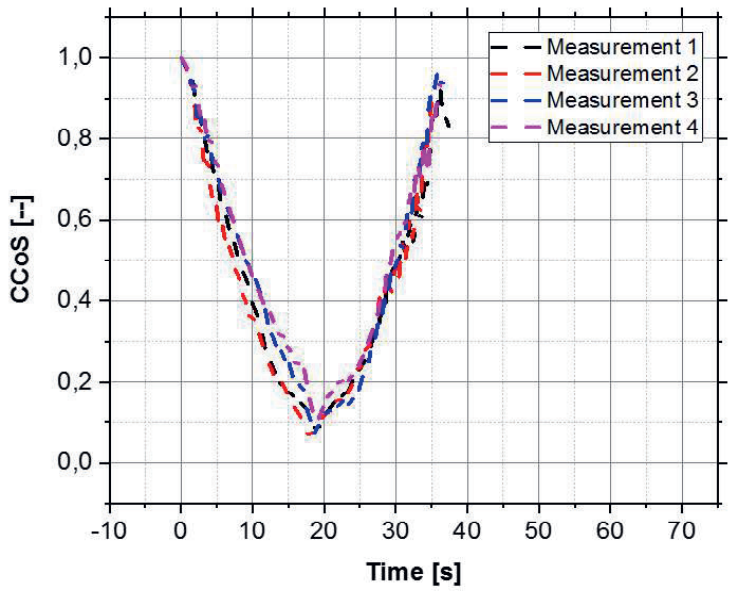

Fig. 7(b): Resulting speckle signal of $\mathrm{Al}_{2} \mathrm{O}_{3}$ sample calculated by equation (2).

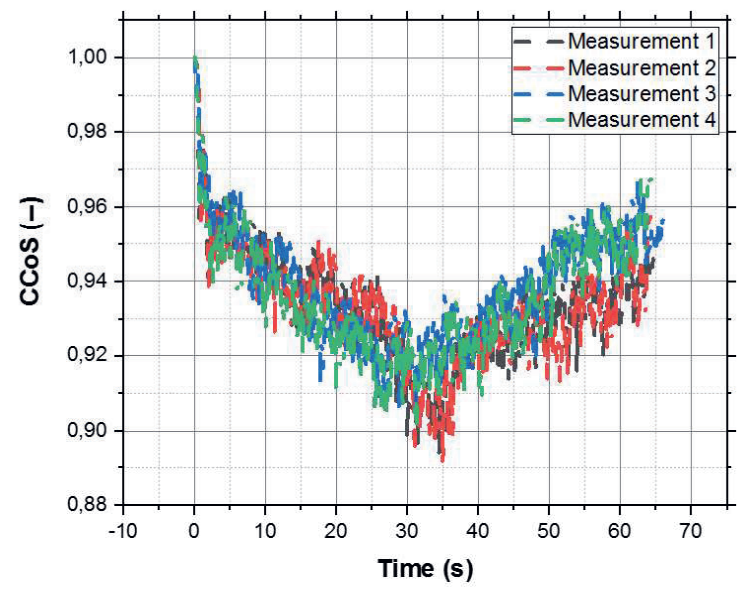

Fig. 7(c): Resulting speckle signal of LTCC sample calculated by equation (2).

From the diagrams, it can be seen that the speckle parameter CCoS has a linear relationship with the mechanical stress introduced by the bending device for both $\mathrm{Al}_{2} \mathrm{O}_{3}$ and LTCC samples in the elastic region. The speckle signals changed direction while the loading condition of bending device turned to unloading. Since the CCoS assesses the similarities of speckle patterns at different stress states, if the speckle patterns change too much, it will lose the correlation to the initial state. In this case, there is a measuring limitation ranging from 0 to circa $90 \mathrm{MPa}$ of $\mathrm{Al}_{2} \mathrm{O}_{3}$ samples at this moment. For LTCC samples, stress differences up to $45 \mathrm{MPa}$ are detectable. However, the measuring range of LTCC is mainly dependent on the strength of the material rather than the correlation range of speckle signal compared with $\mathrm{Al}_{2} \mathrm{O}_{3}$ samples. This phenomenon is caused by different scattering models of these ceramics. In terms of LTCC, it is opaque to the laser at $650 \mathrm{~nm}$ and speckle patterns generated on its surface is determined by surface scattering. On the 
contrary, $\mathrm{Al}_{2} \mathrm{O}_{3}$ is partial transparent to the laser source, and therefore, it has volume scatterings. As we all know, speckle pattern is a result of interference of coherent sources, and the interference is controlled by the differences of optical paths. In surface scattering, surface height fluctuation modulates the optical paths, while in volume scattering it is dominated by the average distribution of path lengths [16], which is caused by the scattering on grain boundaries in the volume. In that case, under the same surface strain condition, the speckle patterns generated by surface scattering changes less than that of volume scattering. From the observations of our experiments, the speckle patterns on the surface of LTCC didn't change so much while the patterns on $\mathrm{Al}_{2} \mathrm{O}_{3}$ changed rapidly and then lost the similarity compared to the speckle pattern at initial stress state. In additional, the noise caused by the camera is around $2 \%$ of CCoS, i.e. 0.02 in numeric value, and it causes the fluctuations in the Fig. 7(c).

\section{Defect detection of ceramics}

The research measurements for defect detection were carried out based on a thermal excitation method. The experimental setup is shown in Fig. 8. Static speckle patterns were generated on sample surface by a laser diode with the wavelength of $650 \mathrm{~nm}$. A camera system including a CMOS chip and an objective worked as a detector to record speckle signal. The camera was focused on sample surface so that the near field subjective speckle patterns were captured. Near field speckle have an interesting feature that their statistical properties are closely related to the structure of the scattering object [17], which means it can be connected to the structure properties of sample surface to provide information of defects for the experiments. The angle between laser diode and camera was approximately $30^{\circ}$. A high power laser with a wavelength of $808 \mathrm{~nm}$ combined with a reflective collimator was used to provide a thermal pulse to excite the sample, which produced dynamic speckle signals. And the pyrometer was a temperature detector. The samples under detection were a $\mathrm{TiO}_{2}$ tube and a Na-ß-Alumina tube. The defects e.g. holes and pores were introduced into sample surface during the manufacturing process. In our measurements, the samples were excited by a $10 \mathrm{~W}$ laser pulse, and the pulse length was around $0.5 \mathrm{~s}$.

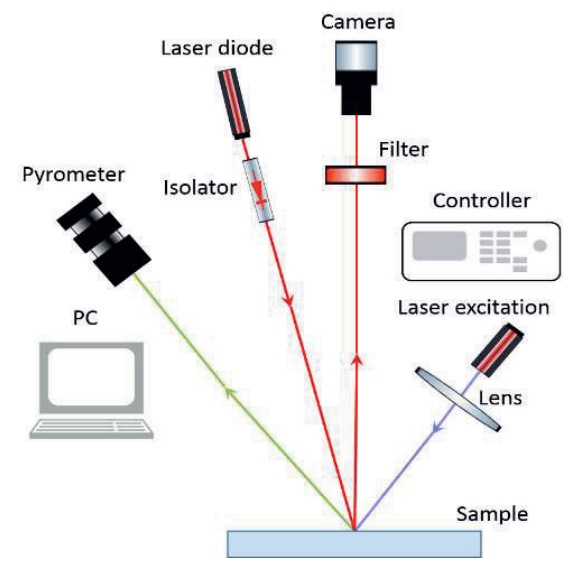

Fig. 8: Experimental setup of LSP for defect detection.
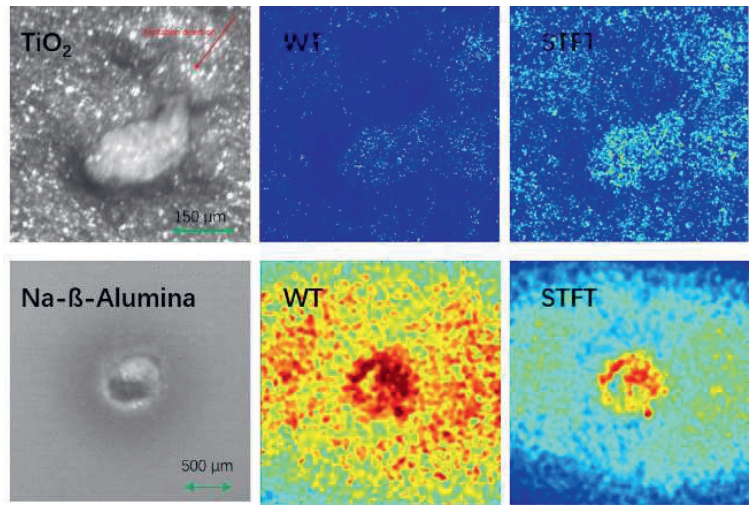

Fig. 9: Resulting images of defect detection. Top: results of $\mathrm{TiO}_{2}$. Bottom: results of Na-ß-Alumina

The preliminary results of current research are shown in Fig. 9. The middle and right parts in Fig. 9 are the results of detection calculated from WT and STFT respectively. And the left parts are the corresponding optical images showing the defects. From the resulting images, it can be observed that the defected are detected by the presence of features with enhanced brightness. And the features show the position and approximate dimension of which correspond well with the defects observed directly in the optical images.

In general, the ideal detectability of LSP is mainly determined by the sensor size of the digital camera, sin the sensor size is far greater than the wavelength of laser source. In reality, at current research the LSP method is constrained by special thermal properties of ceramics, for example, absorption coefficient, thermal diffusivity and coefficient of thermal expansion. These parameters influence the speckle recordings. Extremely, if a material has a small absorption coefficient and a small thermal expansion coefficient, the dynamic speckle movement is difficult to be activated by an external thermal source. In that case, the 
defects cannot be detected due to the lack of speckle information. Meanwhile, the type of defects is also an important factor affecting the detectability. Normally, the LSP technique has a relative higher detectability to sharp defects, because sharp edges give prominence to the effect of thermal accumulation mentioned above. In summary, the detectability of LSP in ceramics is approximately $300 \mu \mathrm{m}$ for crack in length and $200 \mu \mathrm{m}$ for pore and hole in diameter after repeating the measurements with a variety of defective samples in the current research.

\section{Conclusion}

This paper presents an optical sensor system based on the LSP technique for the characterization of ceramics including stress measurement and defect detection. The experimental setups and corresponding evaluation algorithms are proposed. The preliminary results show the possibility for the measurement of stress condition on the surface of ceramic sheet by using specific speckle parameter CCoS. Moreover, LSP has a simple but robust design, reducing cost compared with other conventional optical non-destructive testing methods. And there is a great potential for the LSP technique to develop its detectability in ceramic materials.

\section{Acknowledgments}

The authors would like to thank Federal Ministry for Economic Affairs and Energy for the support within "Zentrales Innovationsprogramm Mittelstand - ZIM - Kooperationsnetzwerke" for the project "SubStress", and FraunhoferGesellschaft for providing the opportunity to participate in the research of Bundesministerium für Wirtschaft und Energie, IGF Forschung project "Entwicklung eines optischen Inline-Verfahrens zur zerstörungsfreien Prüfung keramischer Hochleistungsbauteile". Meanwhile, the authors would like to show the appreciations to all the colleagues in the institutions for the assistance in the investigations.

\section{Reference}

[1] Joseph W. Goodman, [Laser Speckle and Related Phenomena], Springer, New York, chapter 2, 9-75, (1975).

[2] Joseph W. Goodman, [Statistical Optics], Wiley, New York (1985).

[3] U. Cikalova, B. Bendjus, and J. Schreiber. "Laser-Speckle-photometry-A method for noncontact evaluation of material damage, hardness and porosity." Materials Testing 54.2 (2012): 80-84; doi: 10.3139/120.111308

[4] U. Cikalova, B. Bendjus, and J. Schreiber, "Material Characterization by Laser Speckle Photometry", Speckle 2012: V International
Conference on Speckle Metrology (2012); doi: 10.1117/12.978246

[5] U. Cikalova, B. Bendjus, and J. Schreiber, "Laser speckle photometry: contactless nondestructive testing technique." Speckle 2012: V International Conference on Speckle Metrology. Vol. 8413. International Society for Optics and Photonics, 2012; doi: 10.1117/12.978247

[6] S. Muench, et al. "A laser speckle photometry based non-destructive method for measuring stress conditions in direct-copper-bonded ceramics for power electronic application." 2017 18th International Conference on Thermal, Mechanical and Multi-Physics Simulation and Experiments in Microelectronics and Microsystems (EuroSimE). IEEE, 2017; doi: 10.1109/EuroSimE.2017.7926273

[7] D. Y. Tzou, [Macro-to Microscale Heat Transfer: The Lagging Behavior], Second ed. Wiley, 282288 (2014).

[8] Hector J. Rabal, and Roberto A. Braga Jr, eds. [Dynamic laser speckle and applications]. CRC press, 2008.

[9] Robert M. Haralick, "Statistical and structural approaches to texture." Proceedings of the IEEE 67.5 (1979): 786-804; doi: 10.1109/PROC.1979.11328

[10] Ichirou Yamaguchi. "A laser-speckle strain gauge." Journal of Physics E: Scientific Instruments 14.11 (1981): 1270; doi: 10.1088/0022-3735/14/11/012

[11] Zili Sun, Jed S. Lyons, and Stephen R. McNeill. "Measuring microscopic deformations with digital image correlation." Optics and Lasers in Engineering 27.4 (1997): 409-428; doi: 10.1016/S0143-8166(96)00041-3

[12] N. S. Kazak, et al, "Speckle-photometry method of measuring the coefficient of thermal diffusion", Proc. International Conference Optical Techniques and Nano-Tools for Material and Life Sciences, 1, 61 - 72 (2010).

[13] A. Mazzella, A. Mazzella, "The importance of the model choice for experimental semivariogram modeling and its consequence in evaluation process", Journal of Engineering, 2013, 1 - 10 (2013); doi: 10.1155/2013/960105

[14] M. A. Namuq, M. Reich, and S. Bernstein, (2013). "Continuous wavelet transformation: A novel approach for better detection of mud pulses." Journal of Petroleum Science and Engineering, 110, 232-242. doi: 10.1016/j.petrol.2013.08.052

[15] R. X. Gao, and R. Yan, (2006). "Non-stationary signal processing for bearing health monitoring" International Journal of Manufacturing Research, 1(1), 18. doi: 10.1504/ijmr.2006.010701

[16] Joseph W, Goodman, [Speckle phenomena in optics: theory and applications]. Roberts and Company Publishers, 2007.

[17] M. Giglio, et al (2001). "Near-field intensity correlations of scattered light." Applied Optics, 40(24), 4036. doi: 10.1364/ao.40.004036 